\title{
Comparison of Blood Perfusion Parameters in the Ipsilateral and Contralateral Sides of the Brain in Known Cases of Mesial Temporal Lobe Epilepsy
}

\author{
Amin Farzadniya ${ }^{1}$, Jafar Mehvari ${ }^{2}$, Reza Basiratnia ${ }^{2}$ and Farzad Mehrabi ${ }^{1, *}$ \\ ${ }^{1}$ Aja University of Medical Sciences, Tehran, Iran \\ ${ }^{2}$ Isfahan University of Medical Sciences, Isfahan, Iran \\ "Corresponding author: Aja University of Medical Sciences, Tehran, Iran. Email: farzadmehrabi48@ajaums.ac.ir \\ Received 2020 August 02; Revised 2021 May 15; Accepted 2021 May 19.
}

\begin{abstract}
Background: Temporal lobe epilepsy (TLE) is the most common form of focal seizures. To localize the epileptic site for surgery, different neuroimaging tools are used. Perfusion magnetic resonance imaging (MRI) is one of the modalities used to evaluate the cerebral hemodynamics and localize intracranial neoplasia and cerebrovascular events. Two contrast-based perfusion imaging sequences are described, including dynamic susceptibility contrast-enhanced MRI (DSC-MRI) and dynamic contrast-enhanced MRI (DCE-MRI). The most commonly measured parameters include the relative cerebral blood volume (rCBV), relative cerebral blood flow (rCBF), and mean transit time (MTT).

Objectives: This study aimed to evaluate the blood perfusion parameters, such as rCBV and rCBF, in patients diagnosed with mesial temporal lobe epilepsy (MTLE), using DSC-perfusion MRI to determine whether there is a change in these parameters and if this modality can be used to diagnose and localize the epileptic side.

Patients and Methods: Twenty-two patients, who were diagnosed with TLE clinically and electrophysiologically by a neurologist, were investigated in this study. The patients were examined for the presence of any other lesions, such as tumors or cerebrovascular disease as the exclusion criteria. Perfusion images were processed by the Siemens perfusion software, and the rCBV and rCBF maps were generated based on the gamma variate fit. For qualitative analysis, coronal reconstruction of rCBV and rCBF maps was performed. For quantitative analysis, a single neuroradiologist placed the region of interest (ROI) on the hippocampus and the parahippocampal gyrus on T1W images at the same level of DSC images. After determining the CBV and CBF values relative to the ROI of each side, the asymmetry index (AI) was calculated.

Results: In patients with unilateral epilepsy, the blood perfusion parameters in the ipsilateral side of the brain were significantly lower than the contralateral side $(\mathrm{P}<0.0001)$; the mean values of both parameters were significantly lower in the affected side as compared to the opposite side.

Conclusion: In patients with TLE, significantly lower blood perfusion parameters in the affected side of the brain can help radiologists and neurologists to lateralize the MTLE side.
\end{abstract}

Keywords: Perfusion Imaging, CBF, MTLE, CBV, DSC-MR Imaging

\section{Background}

Focal seizures are the most common type of seizures, accounting for almost $60 \%$ of all seizures. The most common form of focal seizures is temporal lobe epilepsy (TLE) (1). TLE was first categorized by the International League Against Epilepsy (ILAE) under the group of localizationrelated symptomatic epilepsy in 1989 (2). There are few epidemiological studies on TLE, and the reports are controversial $(1,3-5)$. TLE is subcategorized as mesial temporal (amygdalohippocampal) and lateral temporal lobe epilepsy (LTLE) or neocortical (6). In 2010, the ILEA identi- fied mesial temporal lobe epilepsy (MTLE) as a subclass of limbic epilepsies. The MTLE was defined as a symptomatic focal epilepsy. The pathophysiology of this condition is usually hippocampal sclerosis (HS) $(2,7)$.

TLE is diagnosed according to the patient's history of focal seizure symptoms and is supported by electroencephalography (EEG), showing a temporal lobe epileptiform-like activity, and by imaging of the lesion (8). Commonly, TLE does not respond well to antiepileptic drugs. It is the most common form of medically intractable epilepsy (MIE), responsible for most epilepsy 
surgeries with a good prognosis (9). To localize the epileptic site for surgery, different neuroimaging methods can be applied. These methods include structural magnetic resonance imaging (MRI) to show the temporal lobe anatomy and the presence of tissue sclerosis, diffusionweighted imaging, diffusion tensor imaging, magnetoencephalography, functional MRI (fMRI), positron emission tomography (PET), and single-photon emission computed tomography (SPECT), all of which can improve the outcomes of surgery $(10,11)$.

Perfusion MRI is one of the modalities used to evaluate the cerebral hemodynamics and localize intracranial neoplasia and cerebrovascular events. Perfusion imaging is divided to non-contrast methods (arterial spin labeling) and contrast-enhanced methods. The contrast-enhanced methods are subdivided into dynamic contrast-enhanced (DCE) T1W imaging and dynamic susceptibility contrast (DSC) T2 * W imaging $(12,13)$. DSC-MRI is the method of choice to measure the brain blood perfusion parameters, as it relies on the $\mathrm{T}^{*}$ signal loss of gadolinium-based contrast bolus, passing through a capillary bed. The most commonly calculated parameters are the relative cerebral blood volume (rCBV), relative cerebral blood flow (rCBF), mean transit time (MTT), and time to peak (TTP) (12-14).

Studies have shown that there may be a significant relationship between TLE and perfusion of the epileptic site. They have investigated different aspects of perfusion changes in epilepsies, especially localized epilepsies, such as TLE (15-21). One of the most inspiring studies was conducted by Xing et al., which demonstrated a decrease in the perfusion parameters in the epileptic side of TLE patients compared to the opposite side (19). However, there are limited studies evaluating changes in the cerebral blood flow perfusion parameters in epileptic patients, and there is no similar study on Iranian patients.

\section{Objectives}

In this study, we aimed to evaluate the blood perfusion parameters, including $\mathrm{rCBV}$ and $\mathrm{rCBF}$, in patients diagnosed with MTLE, using DSC perfusion MRI to determine whether there is a change in these parameters and whether it can be used as a novel alternative method for localizing the epileptic side.

\section{Patients and Methods}

Twenty-two patients (mean age: $26.45 \pm 6.87$ years), who were diagnosed with TLE clinically and electrophysiologically by a neurologist, were studied. All patients had previous MRI scans, indicating unilateral MTLE as sclerosis and volume loss, with no other lesions, such as tumors or cerebrovascular disease (as the exclusion criteria). All patients gave their informed consent about the study procedures, and the ethics committee of the university approved the procedures.

MRI of patients was performed using a 1.5 T MRI scanner (MAGNETOM Aera; Siemens, Erlangen, Germany) and a 12-channel phased-array matrix head coil. The applied protocol included the following sequences: (1) axial T2weighted images (T2WI) with repetition time (TR)/echo time (TE) of $3500 / 125 \mathrm{~ms}$, field of view (FOV) of 230 , and matrix size of $384 \times 256$; (2) axial T1-weighted images (T1W1) with TR/TE of $600 / 26$, FOV of 230 , and matrix size of $320 \times$ 256; coronal 3D fluid attenuated inversion recovery (FLAIR) with TR/TE of 5000/415 ms, inversion time (TI) of $1800 \mathrm{ms,}$ and isotropic pixel size of $0.9 \mathrm{~mm}$; (3) coronal TiW MP-RAGE with TR/TE/TI of 2000/3/1000 ms and 1-mm isotropic pixel size.

The DSC-MRI images were acquired using a $\mathrm{T}^{*}$ weighted gradient-echo, echo-planar imaging (GRE-EPI) sequence with 50 continuous phases. The contrast agent (gadoterate meglumine, DOTAREM) bolus was administered in the 10th phase at $0.2 \mathrm{mmol} / \mathrm{kg}$ body weight dose, followed by $20 \mathrm{~mL}$ of saline flush. The contrast medium was injected by a MEDRAD injector (Liebel-Flarsheim Co., Cincinnati, OH, USA) at an injection rate of $4 \mathrm{~mL} / \mathrm{sec}$. Another axial gradient-echo T1W sequence with the same parameters, such as FOV, slice thickness, and coverage, was obtained after perfusion imaging for a better localization of the region of interest (ROI).

The perfusion images were processed by the Siemens perfusion software, and the rCBV and rCBF maps were generated based on the gamma variate fit. Since the perfusion differences were in the range of $<10 \%$, and the hippocampus could be practically visualized in few axial slices, we used a quantitative analysis. Besides, a coronal reconstruction of the rCBV map in one of the patients in presented in Figure 1.

For the quantitative analysis, a single neuroradiologist with 15 years of experience, who was blinded to the clinical data of the patients, placed the ROI on the hippocampus and the parahippocampal gyrus. The free-shaped ROI with a mean size of 15 pixels was placed on the T1W image, which showed the hippocampus the best, and was copied at the same level of DSC images. After determining the CBV and $\mathrm{CBF}$ values relative to the ROI of each side, the asymmetry index (AI) was calculated by Equation 1(19):

$A I=\frac{100 \times(\text { Contralateral side }- \text { Ipsilateral side })}{\frac{\text { Contralateral side }+ \text { Ipsilateral side }}{2}}$

The DSC MRI-derived perfusion parameters were statistically analyzed in SPSS version 20.0 (IBM, SPSS Inc., USA). 


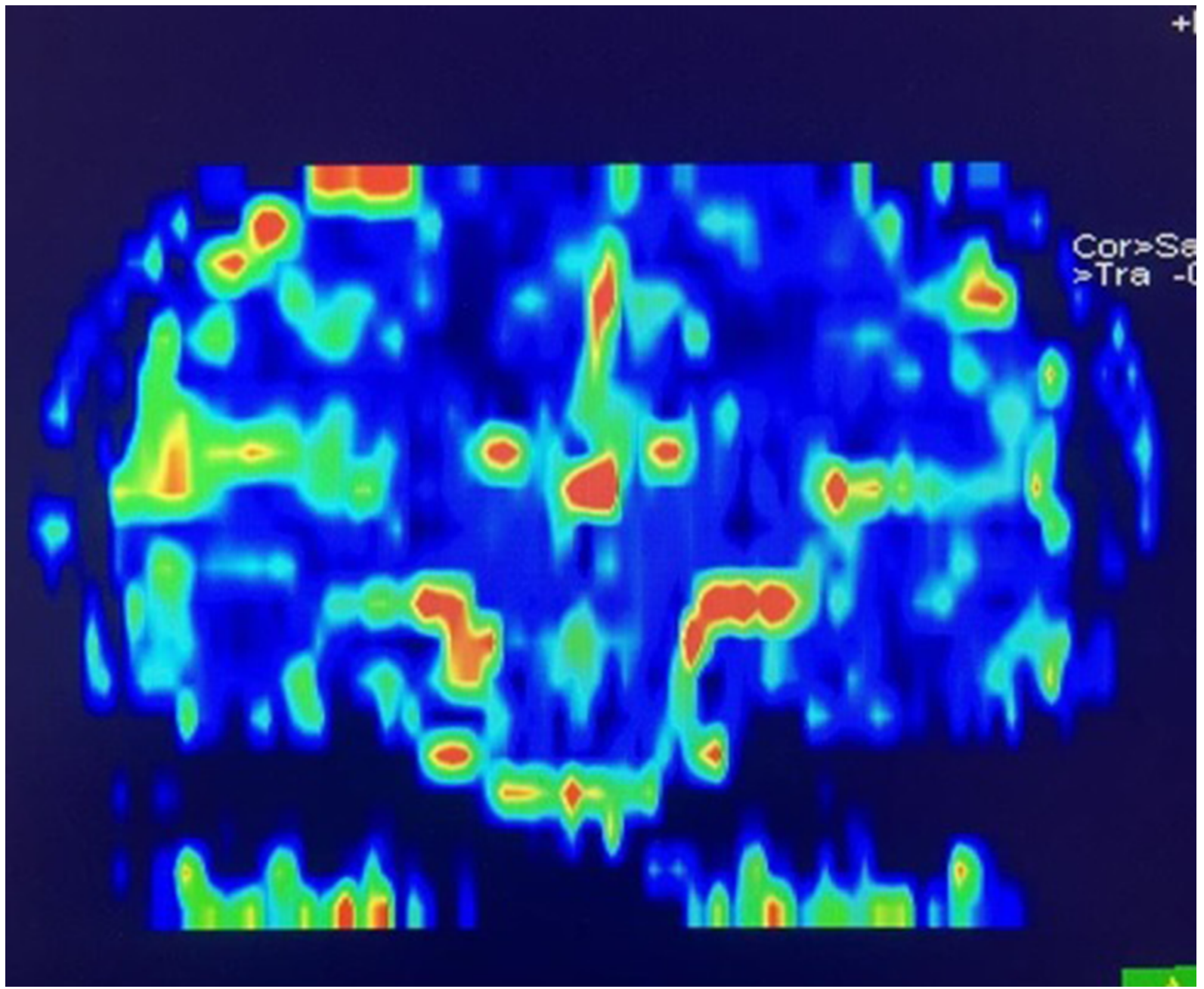

Figure 1. Decreased relative cerebral blood volume (rCBV) on the right side of the hippocampus in patient No. 10 with right-sided temporal lobe epilepsy (TLE).

The normal distribution of parameters in DSC-MRI was assessed using Kolmogorov-Smirnov test. The measured rCBV and rCBF were examined using paired two-sample $t$ tests to determine differences in the perfusion parameters (significance threshold, $\mathrm{P}<0.05$ ).

\section{Results}

A total of 22 patients were enrolled in this study, including 12 (54.4\%) males and 10 (45.5\%) females. Nine patients (40.9\%) had left-sided TLE, and 13 patients (59.1\%) had right-sided TLE. No significant difference was observed in the seizure side between males and females $(\mathrm{P}=0.93)$ (Figure 1). The mean (standard deviation [SD]) age of the participants was 26.45 (6.87) years (range: 16 - 45 years), and the mean (SD) age of the patients with right- and left-sided TLE was 26.23 (7.16) and 26.78 (6.79) years, respectively. No significant difference was found in terms of age between the two groups $(\mathrm{P}=0.85)$. The demographic data of the participants are shown in Table 1.

The results showed that the mean (SD) rCBV was 1.66 (0.29) on the right side (range: 1.24 - 2.21) and 1.88 (0.34) on the left side (range: 1.05 - 2.35) in all patients. Besides, the mean (SD) CBF was 79.30 (16.55) on the right side (range: $51.4-112.3)$ and $89.90(0.21 .61)$ on the left side (range: 41.6 - 118.6). Moreover, the mean (SD) rCBV and rCBF in the affected side (nine left-sided and 13 right-sided) were 1.55 (0.24) and 70.09 (12.59), respectively. For the non-affected side, the mean (SD) values of rCBV and rCBF were 1.99(0.26) and 99.10 (13.57), respectively.

In patients with epilepsy arising from the right side of the brain, the rCBV of the right side was significantly 


\begin{tabular}{|c|c|c|c|c|c|}
\hline Patients & Gender & Age $(y)$ & Epileptic side & AI rCBV & AI rCBF \\
\hline 1 & Male & 18 & Right & 9.76 & 9.93 \\
\hline 2 & Female & 16 & Right & 8.24 & 8.93 \\
\hline 3 & Male & 34 & Right & 9.74 & 10.28 \\
\hline 4 & Female & 21 & Right & 6.22 & 10.46 \\
\hline 5 & Male & 32 & Right & 5.61 & 9.06 \\
\hline 6 & Female & 27 & Right & 8.17 & 17.88 \\
\hline 7 & Female & 25 & Right & 8.16 & 6.99 \\
\hline 8 & Female & 33 & Right & 6.17 & 8.34 \\
\hline 9 & Male & 17 & Right & 11.63 & 10.05 \\
\hline 10 & Male & 38 & Right & 5.46 & 9.85 \\
\hline 11 & Male & 26 & Right & 8.51 & 8.07 \\
\hline 12 & Female & 32 & Right & 5.53 & 8.08 \\
\hline 13 & Male & 22 & Right & 7.73 & 8.37 \\
\hline 14 & Female & 41 & Left & 4.43 & 6.25 \\
\hline 15 & Male & 24 & Left & 2.60 & 7.34 \\
\hline 16 & Male & 28 & Left & 2.52 & 6.61 \\
\hline 17 & Female & 24 & Left & 3.58 & 7.34 \\
\hline 18 & Male & 19 & Left & 5.14 & 12.32 \\
\hline 19 & Female & 19 & Left & 6.43 & 9.06 \\
\hline 20 & Female & 32 & Left & 3.69 & 6.79 \\
\hline 21 & Male & 26 & Left & 3.26 & 5.26 \\
\hline 22 & Male & 28 & Left & 4.03 & 4.54 \\
\hline
\end{tabular}

Abbreviations: AI, assymetry index; rCBV, relative cerebral blood volume; rCBF, relative cerebral blood flow.

lower than the rCBV of the left side $(\mathrm{P}<0.0001)$. Similarly, the right rCBF was lower than the left $\mathrm{rCBF}$, and the difference was significant $(\mathrm{P}<0.0001)$. Overall, the mean values of parameters for the right side of the brain were lower than those of the left side in patients with epilepsy affecting the right side $(\mathrm{P}<0.0001)$. Also, similar analyses were performed in patients with epilepsy of the left side, which indicated similar results $(\mathrm{P}<0.0001)$ (Table 2$)$. In other words, in patients with epilepsy of the left side, both parameters were significantly lower in the left side as compared to the right side. Overall, the mean values of rCBV and rCBF were significantly lower in the affected side compared to the opposite side (Table 3 ).

The AI for both rCBV and rCBF was also measured for all patients listed in Table 1 . The CBV and CBF were significantly different between the two sides of the brain in patients with unilateral epilepsy. Also, the positive values indicated that the parameters were lower in the affected side as compared to the opposite side. Besides, the frequency of epileptic side by gender is shown in Figure 2.

\section{Discussion}

In the present study, 22 patients with MTLE were examined, and the blood perfusion parameters were compared between the involved and healthy sides of the brain hemispheres. The results showed that the rCBV and rCBf were significantly lower in the affected side of patients with TLE, while these parameters were normal in the non-affected cerebral hemisphere. Comparison of the AI values also showed lower blood perfusion parameters in the affected side. Our findings highlighted the association between blood perfusion and TLE.

Some previous studies have investigated the role of brain blood perfusion in patients with epilepsy. In a study by O'Brien et al. in 2007, ten patients with TLE and ten controls were evaluated using MR contrast-enhanced perfusion imaging (MR-CEPI) and diffusion-weighted imaging (DWI). They found that patients with TLE had lower blood perfusion parameters. Based on their results, the CEPI and DWI could be reliable tools for non-lesion TLE (18); these 


\begin{tabular}{|c|c|c|c|}
\hline Variables & Epilepsy of the right side & Epilepsy of the left side & P-value \\
\hline rCBV & & & $\mathrm{P}<0.0001$ \\
\hline Right rCBV & $1.54 \pm 0.20$ & $1.84 \pm 0.31$ & \\
\hline Left rCBV & $2.10 \pm 0.17$ & $1.58 \pm 0.31$ & \\
\hline rCBF & & & $P<0.0001$ \\
\hline Right rCBF & $70.36 \pm 10.38$ & $92.21 \pm 15.49$ & \\
\hline Left rCBF & $103.87 \pm 10.83$ & $69.71 \pm 16.55$ & \\
\hline
\end{tabular}

Abbreviations: $\mathrm{rCBV}$, relative cerebral blood volume; rCBF, relative cerebral blood flow.

${ }^{\mathrm{a}}$ Values are expressed as mean $\pm \mathrm{SD}$.

\begin{tabular}{lccc}
\hline \multicolumn{2}{l}{ Table 3. Comparison of Parameters in the Affected and Non-affected Sides ${ }^{\mathrm{a}}$} & & \\
\hline Variables & Affected side & Non-affected side & P-value \\
\hline rCBV & $1.55 \pm 0.24$ & $1.99 \pm 0.26$ & $\mathrm{P}<0.0001$ \\
CBF & $70.09 \pm 12.59$ & $99.10 \pm 13.57$ & $\mathrm{P}<0.0001$ \\
\hline
\end{tabular}

Abbreviations: rCBV, relative cerebral blood volume; rCBF, relative cerebral blood flow.

${ }^{\text {a }}$ Values are expressed as mean \pm SD.

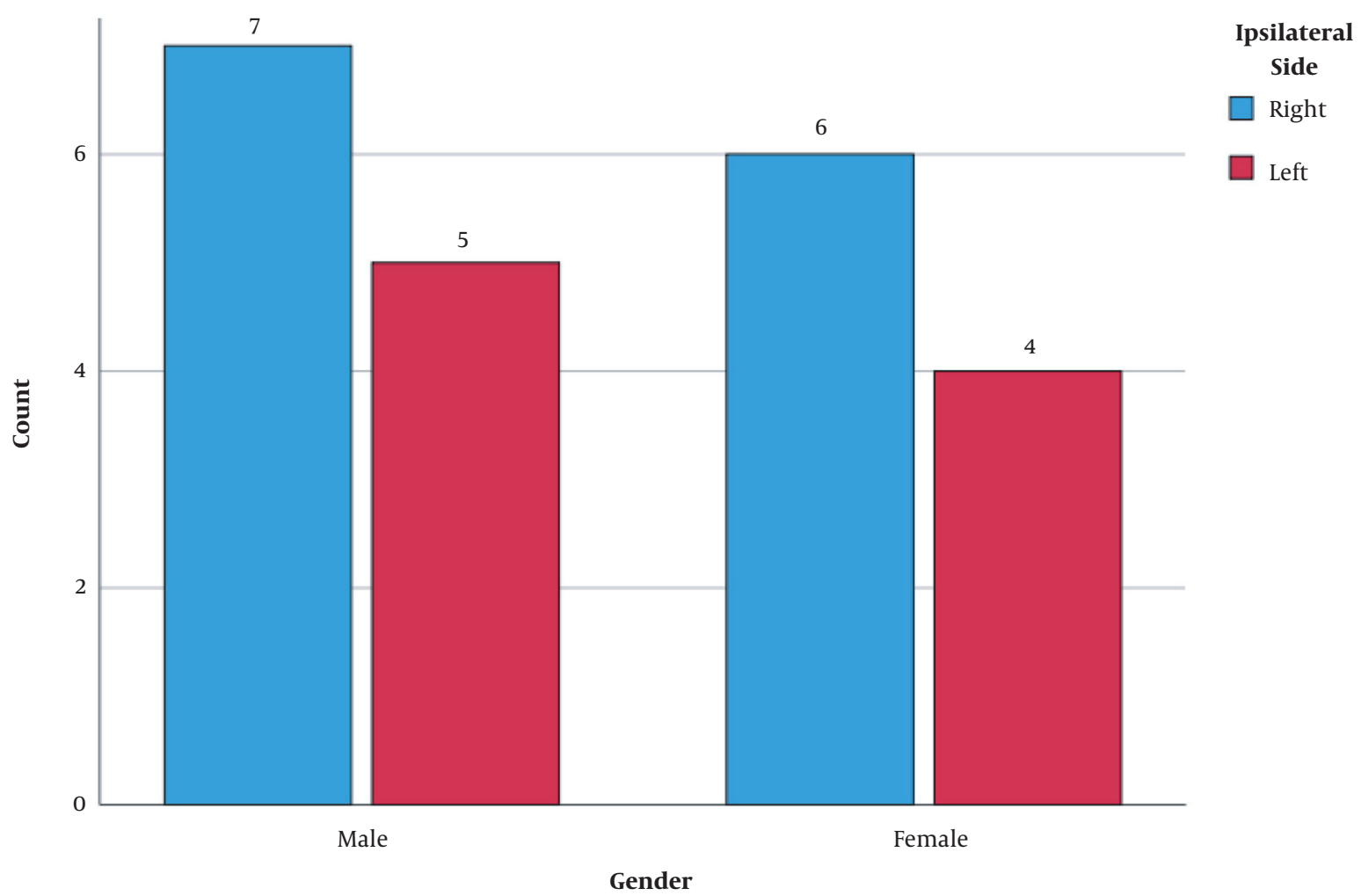

Figure 2. The frequency of epilepsy side in terms of gender 
findings are in line with our results.

In another study by Xing et al. in 2013, a total of 20 interictal cases of TLE and 20 healthy volunteers underwent conventional and DSC MRI. They reported similar results and showed that both rCBF and rCVB were lower in the ipsilateral side, compared to the healthy control subjects. Although our results are similar, both studies lack a large study population. Further research is needed on a larger population, including LTLE patients so that we can strongly recommend DSC-MRI for localizing the epileptic side (19). Moreover, Kim et al. performed a study on 65 patients with intractable mesial temporal epilepsy (MTE). Also, subtraction ictal SPECT co-registered to MRI indicated hypoperfusion in the affected areas of patients with MTE (21).

Moreover, in a study by Oner et al. in 2015, a total of 36 patients with TLE and 11 healthy volunteers were enrolled. Measurements of different parameters showed that in TLE patients, the rCBF ratio of the affected side was significantly lower than the unaffected side. They also emphasized that physicians could localize the affected area in TLE by using rCBF (22); these results are consistent with our findings. However, the key point of our study is that we measured both rCBV and rCBf in the patients and compared the AI values.

In another study by Gelfand et al., changes in the perfusion pattern of the temporal lobe were found in patients with TLE. They also suggested that these abnormalities might be due to atypical vascular distributions; however, further studies are needed in this area (23). Another study by Pizzini et al. from Italy confirmed the effectiveness of pulsed arterial spin labeling (PASL) MRI in diagnosis and lateralization of hypoperfusion in patients with TLE (24). This issue was also discussed by Cleeren et al. in 2015, who showed that different temporal lobe seizures occurred in a common network induced by epileptogenic factors, such as blood perfusion in the brain (25).

The main shortcomings of ASL imaging for TLE localization are the need for a strong magnetic field and limited perfusion parameters that ASL can produce. However, in the present study, by evaluating the difference between parameters in both sides of the brain, we attempted to show that the difference was significant and that DSC-MRI could be applied to localize the epileptic region. We believe that DSC imaging of the brain and comparison of AI values can be reliable tools for a more effective TLE localization, even with a clinical magnetic strength of $1.5 \mathrm{~T}$. The MR perfusion imaging can be used instead of SPECT imaging for tracing physiological changes in the epileptogenic side due to non-ionizing radiation and more spatial resolution.

In conclusion, in patients with TLE, significantly lower blood perfusion parameters in the affected side of the brain can aid radiologists and neurologists to lateralize the MTLE region.

\section{Acknowledgments}

This study was funded by Aja University of Medical Sciences, Tehran, Iran. We would like to express our gratitude to the reviewers for their constructive comments.

\section{Footnotes}

Authors' Contribution: A.F. developed the original idea and protocol of the study, abstracted and analyzed the data, and wrote the manuscript. F.M. contributed to data collection and writing of the manuscript and is a guarantor of this study. J.M. helped collect the data. R.B. contributed to data collection, data analysis, and writing of the manuscript.

Conflict of Interests: There was no conflict of interest.

Ethical Approval: IR.AJAUMS.REC.1398.200.

Funding/Support: This study was funded by AJA University of Medical Sciences.

Informed Consent: All patients gave their informed consent about the study procedures.

\section{References}

1. Tellez-Zenteno JF, Hernandez-Ronquillo L. A review of the epidemiology of temporal lobe epilepsy. Epilepsy Res Treat. 2012;2012:630853. doi: 10.1155/2012/630853. [PubMed: 22957234]. [PubMed Central: PMC3420432].

2. Commission on Classification and Terminology of the International League Against Epilepsy. Proposal for revised classification of epilepsies and epileptic syndromes. Epilepsia.1989;30(4):389-99. doi: 10.1111/j.1528-1157.1989.tb05316.x. [PubMed: 2502382].

3. Semah F, Picot MC, Adam C, Broglin D, Arzimanoglou A, Bazin B, et al. Is the underlying cause of epilepsy a major prognostic factor for recurrence? Neurology. 1998;51(5):1256-62. doi: 10.1212/wnl.51.5.1256. [PubMed: 9818842].

4. Manford M, Hart YM, Sander JW, Shorvon SD. National general practice study of epilepsy (NGPSE): Partial seizure patterns in a general population. Neurology. 1992;42(10):1911-7. doi: 10.1212/wnl.42.10.1911. [PubMed: 1407572].

5. Asadi-Pooya AA, Jowlar H. [The epidemiology and clinical characteristics of temporal lobe epilepsy in South of Iran]. Sadra Med Sci J. 2019;7(3):243-50. Persian.

6. Lau T, Miller T, Klein T, Benbadis SR, Vale FL. Temporal lobe surgery in medically refractory epilepsy: A comparison between populations based on MRI findings. Seizure. 2014;23(1):20-4. doi: 10.1016/j.seizure.2013.09.004. [PubMed: 24094849].

7. Berg AT, Berkovic SF, Brodie MJ, Buchhalter J, Cross JH, van Emde Boas W, et al. Revised terminology and concepts for organization of seizures and epilepsies: Report of the ILAE Commission on classification and terminology, 2005-2009. Epilepsia. 2010;51(4):676-85. doi: 10.1111/j.1528-1167.2010.02522.x. [PubMed: 20196795]. 
8. Sirven JI, Sperling MR, French JA, O'Connor MJ. Significance of simple partial seizures in temporal lobe epilepsy. Epilepsia.1996;37(5):450-4. doi: 10.1111/j.1528-1157.1996.tb00590.x. [PubMed: 8617173].

9. Ramey WL, Martirosyan NL, Lieu CM, Hasham HA, Lemole GJ, Weinand ME. Current management and surgical outcomes of medically intractable epilepsy. Clin Neurol Neurosurg. 2013;115(12):2411-8. doi 10.1016/j.clineuro.2013.09.035. [PubMed: 24169149].

10. Stylianou P, Hoffmann C, Blat I, Harnof S. Neuroimaging for patient selection for medial temporal lobe epilepsy surgery: Part 1 structural neuroimaging. J Clin Neurosci. 2016;23:14-22. doi 10.1016/j.jocn.2015.04.019. [PubMed: 26362835].

11. Stylianou P, Kimchi G, Hoffmann C, Blat I, Harnof S. Neuroimag ing for patient selection for medial temporal lobe epilepsy surgery: Part 2 functional neuroimaging. J Clin Neurosci. 2016;23:23-33. doi: 10.1016/j.jocn.2015.04.031. [PubMed: 26453282].

12. Huisman TA, Sorensen AG. Perfusion-weighted magnetic resonance imaging of the brain: Techniques and application in children. Eur Radiol. 2004;14(1):59-72. doi: 10.1007/s00330-003-1972-y. [PubMed 12827431].

13. Essig M, Nguyen TB, Shiroishi MS, Saake M, Provenzale JM, Enterline DS, et al. Perfusion MRI: The five most frequently asked clinical questions. AJR Am J Roentgenol. 2013;201(3):W495-510. doi: 10.2214/AJR.12.9544. [PubMed: 23971482]. [PubMed Central: PMC3842445].

14. Keston P, Murray AD, Jackson A. Cerebral perfusion imaging using contrast-enhanced MRI. Clin Radiol. 2003;58(7):505-13. doi: 10.1016/s0009-9260(03)00130-2. [PubMed: 12834633].

15. Wolf RL, Alsop DC, Levy-Reis I, Meyer PT, Maldjian JA, GonzalezAtavales J, et al. Detection of mesial temporal lobe hypoperfusion in patients with temporal lobe epilepsy by use of arterial spin labeled perfusion MR imaging. AJNR Am J Neuroradiol. 2001;22(7):133441. [PubMed: 11498422]. [PubMed Central: PMC7975208].

16. Guillon B, Duncan R, Biraben A, Bernard AM, Vignal JP, Chauvel P. Correlation between interictal regional cerebral blood flow and depth-recorded interictal spiking in temporal lobe epilepsy. Epilepsia. 1998;39(1):67-76. doi: 10.1111/j.1528-1157.1998.tb01276.x. [PubMed
9578015].

17. Wu RH, Bruening R, Noachtar S, Arnold S, Berchtenbreiter C, Bartenstein $\mathrm{P}$, et al. MR measurement of regional relative cerebral blood volume in epilepsy. J Magn Reson Imaging. 1999;9(3):43540. doi: 10.1002/(sici)1522-2586(199903)9:3<435::aid-jmri11>3.0.co;2-j. [PubMed: 10194714]

18. O'Brien TJ, David EP, Kilpatrick CJ, Desmond P, Tress B. Contrastenhanced perfusion and diffusion MRI accurately lateralize temporal lobe epilepsy: A pilot study. J Clin Neurosci. 2007;14(9):841-9. doi: 10.1016/j.jocn.2006.07.003. [PubMed:17596947].

19. Xing W, Wang X, Xie F, Liao W. Application of dynamic susceptibility contrast-enhanced perfusion in temporal lobe epilepsy. Acta Radiol. 2013;54(1):107-12. doi: 10.1258/ar.2012.110658. [PubMed: 23117196].

20. Nelissen N, Van Paesschen W, Baete K, Van Laere K, Palmini A, Van Billoen $\mathrm{H}$, et al. Correlations of interictal FDG-PET metabolism and ictal SPECT perfusion changes in human temporal lobe epilepsy with hippocampal sclerosis. Neuroimage. 2006;32(2):684-95. doi: 10.1016/j.neuroimage.2006.04.185. [PubMed:16762567].

21. Kim BJ, Hong SB, Seo DW. Differences in ictal hyperperfusion of limbic-related structures between mesial temporal and neocortical epilepsy. Epilepsy Res. 2008;81(2-3):167-75. doi: 10.1016/j.eplepsyres.2008.05.010. [PubMed:18639441].

22. Oner AY, Eryurt B, Ucar M, Capraz I, Kurt G, Bilir E, et al. pASL versus DSC perfusion MRI in lateralizing temporal lobe epilepsy. Acta Radiol. 2015;56(4):477-81. doi: 10.1177/0284185114531128. [PubMed: 24782571].

23. Gelfand JM, Wintermark M, Josephson SA. Cerebral perfusion-CT patterns following seizure. Eur J Neurol. 2010;17(4):594-601. doi: 10.1111/j.1468-1331.2009.02869.x. [PubMed: 19968701].

24. Pizzini FB, Farace P, Manganotti P, Zoccatelli G, Bongiovanni LG, Golay X, et al. Cerebral perfusion alterations in epileptic patients during peri-ictal and post-ictal phase: PASL vs DSC-MRI. Magn Reson Imaging. 2013;31(6):1001-5. doi: 10.1016/j.mri.2013.03.023. [PubMed: 23623332].

25. Cleeren E, Casteels C, Goffin K, Janssen P, Van Paesschen W. Ictal perfusion changes associated with seizure progression in the amygdala kindling model in the rhesus monkey. Epilepsia. 2015;56(9):1366-75. doi: 10.1111/epi.13077. [PubMed: 26174547]. 